\section{Las percepciones del tutor clínico sobre su desempeño docente: un estudio reflexivo}

\author{
PATRICIA PALMA ${ }^{1}$, CAROLINA WILLIAMS ${ }^{1, a}$, LUCÍA SANTELICES ${ }^{1, b}$
}

\section{Tutorial teaching in medicine from the perspective of teachers}

Background: During clinical tutoring, medical students are trained to transfer theoretical knowledge to real life situations that need to be solved. Tutors should be trained to acquire skills to encourage motivation, transfer experience and inspire interest in learning among students. Aim: To inquire about interests and needs during the daily work of clinical tutors. Material and Methods: A qualitative study inserted in the Grounded Theory, based on the description of the meanings, in which ten clinical tutors responded to an interview in their own workplace. After obtaining consent, the responses were recorded and transcribed using Georgi's method for analysis. Results: Teachers feel that there is a gap between the theoretical basis of tutorial teaching and the reality. They are especially interested in a teaching process based on values. They recognize that their pedagogical skills should be improved to improve their communication with students. They also feel that they require more skills to transfer theoretical knowledge to clinical situations. Conclusions: A personal satisfaction for educating and training, the transmission of experiences, empathy and being assertive are emerging values that motivate tutors to deliver a quality education, above remunerations or contractual ties. However, the skills to transfer clinical knowledge to students should be improved.

(Rev Med Chile 2020; 148: 535-541)

Key words: Grounded Theory; Medical Education; Perception; Qualitative Research.
'Oficina de Educación Médica,

Facultad de Medicina,

Universidad Finis Terrae.

Santiago, Chile.

aProfesora, Magíster en Docencia Universitaria en Ciencias de la

Salud.

bProfesora, Magíster en

Educación Especial.

Trabajo no recibió financiamiento. Los autores declaran no tener conflictos de interés.

Recibido el 17 de diciembre de 2019, aceptado el 5 de mayo de 2020.

Correspondencia a: Carolina Williams Avenida Pedro de Valdivia 1509, Providencia. Santiago, Chile. cwilliams@uft.cl

\section{A} partir del análisis realizado por el Espacio Europeo en Educación Superior (EEES), se suscitaron numerosos cambios en el paradigma docente centrado en el aprendizaje, cobrando mayor importancia la función mediadora y tutorial del profesor universitario. Este cambio en los roles de estudiantes y sobre todo en el rol docente ha condicionado en forma directa la formación teórica y práctica impactando de manera muy especial en las competencias declaradas en los perfiles de egreso en carreras de ciencias de la salud $^{1-3}$. Es en este contexto, donde la docencia realizada en los campos clínicos toma mayor protagonismo, porque, es función del tutor, potenciar las competencias profesionales enunciadas en el perfil de egreso de los estudiantes, tales como, sólidos conocimientos en ciencias médicas, capacidades para atender y resolver patologías junto a un espíritu de servicio, actitud crítica, alto grado de reflexión y autonomía, todos elementos que se fortalecen en la tutoría clínica durante la práctica profesional.

La tutoría clínica es una actividad pedagógica que tiene como uno de sus propósitos formar, orientar, y apoyar a los estudiantes durante su proceso formativo, transfiriendo conocimientos adquiridos en el aula a la solución de problemas y necesidades clínicas ${ }^{4}$. La tutoría clínica se defi- 
ne como una actividad académica donde ocurre transferencia de conocimiento disciplinar a la parte clínica de manera informada, disciplinada y creativa. Esta actividad académica incluye declarar objetivos claros y estimular el razonamiento clínico, pensamiento crítico y la metacognición ${ }^{4,5}$.

La enseñanza en el entorno clínico es una tarea exigente, compleja y a menudo, desmotivante. Una tarea que muchos clínicos asumen sin una adecuada preparación u orientación pedagógica que puede generar en los estudiantes experiencias frustrantes. Muchas veces, se replican modelos tradicionales de docencia y se realiza la actividad tutorial de forma intuitiva. A esta realidad se le suman las exigencias de aprendizaje centrado en el estudiante, que requiere nuevos métodos de enseñanza más orientados al aprendizaje, métodos de evaluación basados en competencias y el énfasis en el profesionalismo, lo que exige que los docentes clínicos cuenten no solo, con una sólida experiencia clínica sino con una amplia gama de herramientas pedagógicas ${ }^{6,7}$. $\mathrm{Al}$ respecto, muchas investigaciones coinciden en la necesidad de formar docentes clínicos competentes, con manejo de un modelo pedagógico para abordar las tutorías clínicas y con herramientas educativas que les permita conducir su docencia en forma reflexiva, para ayudar a los estudiantes a explorar distintas alternativas en el manejo de problemas asociados con el aprendizaje en clínica ${ }^{4,8}$.

Con el fin de apoyar a los docentes en los escenarios clínicos, la mayoría de las escuelas de medicina ofrecen una variedad de programas de perfeccionamiento orientados a mejorar las competencias requeridas por los médicos que ejercen docencia clínica ${ }^{9-12}$. Sin embargo, los programas ofrecidos no necesariamente se abordan considerando la realidad especifica del contexto clínico, ni los intereses y necesidades reales experimentadas por el tutor clínico en su trabajo cotidiano.

El propósito del siguiente estudio es indagar sobre la percepción que el tutor tiene acerca de sus intereses y necesidades reales como tutor clínico en su trabajo cotidiano y aportar evidencia para ajustar la formación de los tutores.

\section{Material y Método}

El presente estudio se enmarca en la metodología cualitativa, se inserta en la Teoría Fundamen- tada, a partir de la descripción de los significados vividos y experimentados por cada uno de los participantes. El estudio se llevó a cabo durante un semestre en el consultorio universitario Santa Anselma de atención primaria de la Universidad Finis Terrae.

La muestra se conformó por 10 tutores clínicos que se sometieron a una entrevista en su propio lugar de trabajo, (2 ginecólogos, 2 médicos internistas, 2 neurólogos y 4 pediatras). El instrumento utilizado para recopilar la información corresponde a una entrevista semiestructurada. Se elaboró previamente un guion temático incluyendo indicadores teóricos considerados relevantes en la docencia clínica y que son reportados por: Harden en el año 2000; Hesketh durante el 2001, Ramani y McLean en el 2008 y Triviño en el año 2009 $9^{13-17}$, se gestaron cuatro categorías:1) Buen desempeño docente; 2) Profesionalismo; 3 ) Gestor y facilitador del aprendizaje y 4) Desarrollo profesional en la enseñanza médica.

El guión temático consideró 17 preguntas abiertas (Anexo 1). La entrevista tuvo una duración entre 45 y $60 \mathrm{~min}$. Se realizó en el box de cada médico de manera privada y sin interrupción en un ambiente de respeto y confianza adecuándose la investigadora al horario del profesional. La entrevista se grabó para luego transcribirse.

Para el análisis de los datos del estudio se utilizó el método Giorgi ${ }^{18}$.

Con el fin de asegurar el rigor científico del estudio se procedió a validar el guion temático de la entrevista por juicio de expertos. También se validó con el sujeto entrevistado, las interpretaciones realizadas por el investigador. La reducción de los datos se estableció hasta alcanzar la saturación de los mismos.

La investigación contó con la aprobación del comité de ética de la Universidad y con el consentimiento informado de cada uno de los participantes.

\section{Resultados}

8 son las categorías que muestran un alto significado para los tutores clínicos entrevistados. 1) Cualidades del tutor; 2) Modelaje en tutoría clínica; 3) Metodología de enseñanza basada en la intuición y la experiencia; 4) Programa y planifica- 
ción de la docencia; 5) estrategias para favorecer el aprendizaje; 6) Perfeccionamiento docente; 7) retroalimentación sobre el desempeño docente y 8 ) entusiasmo y motivación por la docencia (código emergente) (Tabla 1).

\section{Categoría: Cualidades del tutor}

Las principales cualidades en las que coinciden la mayoría de los tutores son paciencia, empatía, ser accesible y ser sensible a las necesidades de aprendizaje de cada estudiante, el tutor debe tener conocimientos sólidos y valóricos. Si bien ellos destacan entre las cualidades del tutor aspectos personales que muestran la valoración que ellos tienen por esta tarea, llama la atención que en sus discursos no incorporan las habilidades cognitivas y comunicativas que les permiten realizar su trabajo tutorial.

\section{Tabla 1. Proposiciones significativas que emergen desde el discurso del tutor sobre su rol como} docente clínico

\begin{tabular}{|c|c|}
\hline Categoría & Fragmento de discurso ilustrativo \\
\hline \multirow[t]{4}{*}{ 1. Cualidades del tutor } & $\begin{array}{l}\text { "Yo creo que el tutor clínico debería buscarse básicamente no por su capacidad académica úni- } \\
\text { camente, porque en los médicos yo veo que hay gente tremendamente capaz, pero tienen una } \\
\text { incomunicación tremenda con los alumnos y con la gente" (T5: 232-236) }\end{array}$ \\
\hline & $\begin{array}{l}\text { "Como profesionales de la salud también son fundamentales estas bases digamos valóricas } \\
\text { que nos van a permitir dar un buen desempeño y buen ejemplo para ellos, pero sin que signi- } \\
\text { fique menos que el tema de conocimientos sólidos y básicos y constante perfeccionamiento es } \\
\text { importante" (T4: 253-259) }\end{array}$ \\
\hline & $\begin{array}{l}\text { "Primero yo creo que tiene que tener paciencia, tener tolerancia también, cierto, yo creo que } \\
\text { debe tener buen carácter amplitud de criterio" (T6: 365-373). }\end{array}$ \\
\hline & Ser empático, tener una cercanía con el alumno" (T7: 279) \\
\hline \multirow[t]{2}{*}{$\begin{array}{l}\text { 2. Modelaje en tutoría } \\
\text { clínica }\end{array}$} & $\begin{array}{l}\text { "Es muy importante porque los alumnos nos ven a nosotros como modelos, modelos a seguir, } \\
\text { es importante enseñarle al alumno que no está tratando a un órgano, no está tratando a una } \\
\text { enfermedad, está tratando a un paciente, está tratando a una persona que tiene una enferme- } \\
\text { dad y todo el trabajo que hacemos tiene que estar orientado hacia la persona, no solamente } \\
\text { curar su enfermedad y esa persona es un alguien que viene con muchas variables a parte de su } \\
\text { enfermedad" (T10: 33-38) }\end{array}$ \\
\hline & $\begin{array}{l}\text { "Lo dividiría como en dos etapas uno características de uno como persona, somos modelos } \\
\text { a seguir de ellos, el cómo saludamos al paciente, de cómo le tratamos, como les explicamos } \\
\text { su patología, a si acogemos sus dudas, ser receptivo con el paciente súper importante porque } \\
\text { ellos aprenden como actúa mi tutor, como yo quiero ser y segundo en cuanto a conocimientos, } \\
\text { eeehh si bien tener todas las bases sólidas para darles formación, también está el hecho de ser } \\
\text { abierto y no cerrado" (T5: } 30-33 \text { ) }\end{array}$ \\
\hline \multirow{3}{*}{$\begin{array}{l}\text { 3. Metodología de } \\
\text { enseñanza y aprendizaje } \\
\text { basada en la intuición y } \\
\text { la experiencia personal }\end{array}$} & $\begin{array}{l}\text { "Yo no tengo una educación pedagógica, yo tengo la experiencia de mis propios profesores y } \\
\text { lo que yo sentí cuando estudiaba la carrera y que falencias veía, que me hubiese gustado a mí } \\
\text { que me dijeran o que me hicieran ver en ese momento".(T1: 400-403) }\end{array}$ \\
\hline & $\begin{array}{l}\text { "Yo creo que, que es lo que haría yo, que fue lo que hicieron los maestros, buscar alguna manera } \\
\text { profesional de incluir alguna conversación, seminario o taller en forma paralela a lo estrictamente } \\
\text { técnico de cosas más humanistas, yo a todos los internos les hablo de ópera, de arte, de pintura, } \\
\text { de escritura, de que hace la polola, que hacen tus papás, siempre me doy el tiempo y creo que } \\
\text { esa cuestión te permite comunicarte mejor" (T4: 253-259) }\end{array}$ \\
\hline & $\begin{array}{l}\text { No, no, no tengo formación pedagógica, sólo la experiencia de la práctica...". "A veces uno hace } \\
\text { cosas por intuición y con la mejor de las intenciones." (T7: 292) }\end{array}$ \\
\hline \multirow{2}{*}{$\begin{array}{l}\text { 4. Programa y } \\
\text { planificación de la } \\
\text { docencia }\end{array}$} & $\begin{array}{l}\text { "El programa si, generalmente nos lo envían, no te voy a decir que lo he leído en profundidad } \\
\text { y todo completo, pero en general los objetivos como a nivel global están..." (T1: 305-309) }\end{array}$ \\
\hline & "Lo he leído, Si, pero no ha gran detalle, pero sí lo conozco" (T4: 186) \\
\hline
\end{tabular}




\section{Tabla 1. Proposiciones significativas que emergen desde el discurso del tutor sobre su rol como} docente clínico

\section{Categoría}

5. Estrategias metodológicas utilizadas para el aprendizaje.
6. Perfeccionamiento docente

\section{Fragmento de discurso ilustrativo}

“Estrategia eehhmmm, haber es que en los talleres ocupo varias modalidades dependiendo del taller, por ejemplo hay taller que es el primero que hacemos que es de anamnesis en que es un juego de roles, después hay otro que es con casos clínicos, o sea son casos clínicos en que ellos tienen que empezar con la anamnesis, motivo de consulta, anamnesis próxima, anamnesis remota, pero enfocada al caso clínico y llegar a un diagnóstico, una hipótesis diagnóstica, hay otro momento que por ejemplo escuchamos ruidos respiratorios y lo empezamos aahhmmm digamos analizar y definir" (T9: 111-133)

"Empecé hacer seminarios reforzando las cosas porque cuando uno les hace un seminario al otro día aparece un paciente con los mismo y entonces te acuerdas de ayer y vimos esto, entonces los seminarios sirven mucho pa para reforzar la materia y después aplicarla y les queda, les queda bastante más fácil que cuando cada uno sabe lo suyo, como que unificas términos o unificas definiciones y eso hace que después solito les caiga la teja, medio coloquial salió el término" (T8: 83-89)

"Me gustaría perfeccionar el tema de la evaluación, mucha vez pasa que uno es muy subjetivo, entonces claro una forma que sea más concreto o más objetivo a de evaluar".

"No, pero me encantaría tenerla, los ubico, los conozco, pero no me los he podido pagar" (T8: 209-212)

"No, como pedagogía como tal no, así como cursos no, no" (T7: 252-255)

"No no tengo una formación formal de hacer un diplomado o algo en educación" (T6: 336-341)

"Es importantísimo claro porque uno sabe trabajar como médico, pero no sabe el trabajo como docente, nunca nadie te enseño hacer un docente uno sigue el modelo de cómo te enseñaron no más".

"Los estudiantes siempre, ahí ellos siempre te hacen saber si les gusta o no les gusta lo que uno está haciendo" "me gustaría que me retroalimentaran, Siiii, de todas maneras, porque así uno sabe si lo está haciendo bien o no" (T4: 202-204)

7. Retroalimentación sobre el desempeño docente

"Si en general, que bien que, por la parte humana, porque en el fondo ellos no me están evaluando directamente que hablo, que digo, sino que en el fondo es como la opinión general de los chiquillos como ellos te ven desempeñando tu trabajo" (T2: 349-351)

"Para mí lo más importante es que los chiquillos tengan una visión más integral..., entonces a mí, me interesa, la prevención, la promoción, no todo es saberse el libro al revés y al derecho." (T8: 26-28)

8. Entusiasmo y motivación por la docencia

"Es muy gratificante poder enseñar algo a una persona y que uno llegue a ser una diferencia en el camino de esa persona en cuanto a su formación académica" (T10:15-17)

"En la medida que uno va enseñando siempre va aprendiendo cosas nuevas, actualizándose de alguna forma, hace que uno se cuestione lo que actualmente se sabe y genere nuevas preguntas de investigación" (T10:20-23)

"Me gusta harto el trabajo de docente, me gusta la cercanía con los alumnos" (T3: 16)

Está el tema de, primero la motivación el interés por la docencia, motivación y el interés de contribuir a reforzar las cosas que uno considera que están en déficit en los chiquillos" (T7: 31-34)

"Amo la docencia, amo enseñar, uno aprende " $N$ ", no solamente enseña, aprende mucho con los estudiantes" (T8: 23/24)

(Tn:xx-xx): Número tutor; líneas de transcripción representativas del discurso. 


\section{Categoría: Modelaje en tutoría clínica}

El rol de modelo es esencial para establecer una buena relación médico-paciente, y para formar médicos con un enfoque integral. Los discursos que se levantan desde los tutores clínicos confirman la alta valoración que ellos tienen de su función como modelos de sus estudiantes.

Los discursos de los tutores no refieren explícitamente otros roles que les son propios, tales como: las formas de abordar al paciente para recoger las evidencias, razonar clínicamente a partir de la información recabada durante la interacción y como ellos transfieren el conocimiento teórico al conocimiento práctico en función de cada paciente.

\section{Categoría: Metodología de enseñanza y aprendizaje basada en la intuición y la experiencia personal}

Los tutores entrevistados reconocen que la metodología que aplican es en base a la intuición y propia experiencia. Enfatizan su interés por la formación de sus estudiantes y se responsabilizan afectivamente de ésta. Al referirse a las formas de llevar a cabo la docencia se centran los discursos en replicar su propia experiencia, no aparecen nociones de estrategias pedagógicas para fortalecerla.

\section{Categoría: Programa y planificación de la docencia}

Los tutores ponen en evidencia que, aun conociendo la importancia de contar con un programa de curso, ellos no lo consideran para su docencia. Atribuyen lo señalado a la falta de tiempo con que cuentan para realizar su trabajo. Este hecho implica repercusiones negativas para la formación de los estudiantes y resultados de aprendizajes aleatorios.

\section{Estrategias metodológicas utilizadas para el aprendizaje}

Para facilitar el aprendizaje de sus estudiantes los tutores utilizan recursos como los seminarios, talleres, role playing, preguntas abiertas, lluvias de ideas, análisis de casos clínicos y guías de autorregulación. Los discursos mencionan técnicas de enseñanzas siempre asociándolas con el contenido no se menciona la estimulación de habilidades clínicas.

\section{Categoría: Perfeccionamiento docente}

Los tutores participantes están conscientes de la falta de herramientas pedagógicas y de la necesidad de perfeccionarse, sus discursos denotan motivación e interés por realizar perfeccionamiento, sin embargo, se observa un vacío acerca de la conciencia de trabajar pedagógicamente con la estimulación de habilidades relacionadas con el razonamiento clínico. Los tutores tienen la motivación por hacer un trabajo educativo eficiente, sin embargo, la falta de tiempo y los costos están presentes al momento de realizar algún programa de capacitación en docencia tutorial.

\section{Categoría: Retroalimentación sobre el desempeño docente}

Para los tutores es necesario ser retroalimentados constantemente sobre su desempeño ya sea de parte de las autoridades de la Universidad como de sus propios estudiantes, con el fin de orientar su trabajo y saber qué pasos dar para mejorar. La necesidad de logro se vería compensada si los tutores que se enfrentan a una tarea de naturaleza compleja recibieran la retroalimentación de sus autoridades respecto de su trabajo. Con ello se daría satisfacción a su necesidad de logro y de afiliación.

\section{Categoría: Entusiasmo y motivación por la docencia (código emergente)}

Transmitir los conocimientos con un compromiso en la formación integral del estudiante. Se repite mucho el término "formar médicos más humanos". El análisis de discurso realizado permite comprender que el interés por la docencia y los resultados positivos que el tutor recoge contribuyen a reforzar su motivación intrínseca que va en beneficio de los estudiantes, de querer hacer bien su tarea. Los tutores demuestran alta motivación por la tarea docente.

\section{Discusión}

El siguiente estudio pone en evidencia factores que generan una brecha entre el estándar de desempeño docente que se propone desde la evidencia teórica y la realidad que afronta un tutor clínico.

Desde la evidencia teórica recabada por trabajos como el de Ramani 2008, Harden 2000, Hesketh 2001, así como la revisión hecha por Triviño $\mathrm{X}, 2009$, se levantaron indicadores de competencia para la enseñanza de campo clínico agrupados en 4 categorías; desempeño docente, profesionalismo, gestión del aprendizaje y desarrollo profesional en 
la enseñanza médica. Sí bien todas estas categorías con sus propios indicadores levantados desde la evidencia teórica perfilan el desempeño óptimo de un docente clínico, pudimos constatar que se abordan desde una realidad que es ajena al tutor clínico, el cual se ve enfrentado diariamente a un escenario diverso, complejo y cambiante que interfiere de cierta manera en el desarrollo de la sesión clínica, no obstante, para el tutor clínico el valor y el sentido que tiene su labor docente transciende lo cotidiano y requiere herramientas pedagógicas ${ }^{1}$. Los resultados confirman lo señalado en estudios recientes, si bien, los tutores valoran positivamente su acción docente, reconocen la necesidad de acceder a herramientas pedagógicas que hagan su labor más fructífera ${ }^{2}$.

Creemos que es desde la actitud positiva y su nivel de satisfacción desde donde se debe abordar la formación pedagógica de los tutores clínicos, considerando los aspectos actitudinales y vivenciales que ellos experimentan en su campo clínico, sobre todo el valor que le dan a la motivación por la docencia, la validación del rol del tutor, la relación de cercanía y empatía con sus estudiantes, así como también al "llamado vocacional" que la mayoría sienten por su labor docente.

Pensamos que estamos frente a una "fuente vocacional" que se debe potenciar con una capacitación dirigida desde aquello que los mueve, les hace sentido y con un enfoque desde su realidad.
Son los propios tutores quienes reconocen que no basta con la experiencia y la experticia que tengan en relación a la disciplina que guían, están conscientes de que necesitan herramientas pedagógicas sobre todo en métodos de evaluación, en planificación, en metodología de enseñanza-aprendizaje y métodos de comunicación efectiva ${ }^{19}$. Para esto los tutores solicitan tiempo protegido para las capacitaciones y financiamiento para realizarlas.

Por otra parte, los tutores expresan su necesidad de ser constantemente retroalimentados de manera formal sobre su desempeño, no solo, de parte de sus estudiantes, sino también de parte de las autoridades de la Universidad porque esto les permite ser más objetivos y críticos con respecto a su desempeño y procurar mejoras ${ }^{20}$.La evidencia teórica propone un estándar "de calidad", "de buen desempeño docente", que es la meta a la que todos los docentes clínicos deberían llegar, sin embargo, esta meta debe ser con una mirada aterrizada sobre la realidad del tutor, sobre su escenario clínico y las dificultades que enfrentan a diario, considerando todos los aspectos actitudinales que ponen de manifiesto en su labor docente ${ }^{3}$.

Agradecimientos: Agradecemos a las Escuela de Medicina de la Facultad de Medicina de la Universidad Finis Terrae, en especial a su director Ernesto Vega Asún por apoyar el desarrollo de este trabajo.

\section{Anexo 1. Guión temático de la entrevista}

1. ¿Qué impresión tienes acerca de la necesidad de planificar cada sesión práctica?

2. ¿Qué apreciación tienes de que en cada sesión práctica se deben dar a conocer los objetivos y metas antes del desarrollo de la actividad clínica?

3. Con respecto a tu desempeño docente ¿cómo facilitas el clima de aprendizaje positivo y autónomo de tus estudiantes? ¿puedes mencionar de qué manera lo haces?

4. ¿Qué sentido tiene para ti el rol del tutor clínico como modelo, guía y orientador del proceso de aprendizaje de los estudiantes?

5. ¿Qué características consideras que son importantes para ejercer como tutor clínico?

6. ¿Según la forma en que concibes la tutoría clínica, cuáles serían los atributos que debe tener un tutor clínico, para desempeñarse como tal?

7Conoces el programa de estudio de tu asignatura y como se articula con otras disciplinas?

8. ¿Qué estrategias pedagógicas utilizas para facilitar el proceso de enseñanza-aprendizaje de tus estudiantes?

9. ¿En tu ejercicio como tutor clínico como estimulas el razonamiento clínico?

10. ¿Tienes algún nivel de formación pedagógica?, ¿Cuál y dónde lo obtuviste?

11. ¿Conoces algún modelo sistemático de formación en tutoría clínica?

12. ¿En tu desempeño como tutor clínico qué importancia tiene el perfeccionamiento en el área docente?

13. ¿Qué área o ámbito de tu trabajo con los estudiantes te gustaría perfeccionar mediante cursos de educación médica?

14. ¿Has recibido alguna vez retroalimentación acerca de tu desempeño docente como tutor clínico?, ¿sientes que es necesario?

15. ¿Cuáles fueron tus motivos para ser tutor clínico?

16. ¿Qué importancia tiene para ti la tutoría clínica?

17. En lo que respecta a la importancia de guiar el proceso de aprendizaje de los estudiantes en el campo clínico. ¿Puedes contar alguna experiencia, situación, o momento que hayas tenido con algún estudiante y que te haya marcado positivamente? 


\section{Referencias}

1. González J, Wagenaar R. Una introducción a Tuning Educational Structures in Europe. La contribución de las universidades al proceso de Bolonia. Bilbao Publicaciones la Universidad Deusto. 2009; 97p.

2. King E, Turpin M, Green W, et al. Aprender a interactuar e interactuar para aprender: una teoría sustantiva del aprendizaje clínico en el lugar de trabajo para diversas cohortes. Adv en Salud Sci Educ 2019; 24: 691-706.

3. Álvarez P, González M. Analysis and conceptual assessment on the modalities of university tutoring in the European Higher Education Area. Rev Interuniversitaria Formación del Profesorado 2008; 22 (1): 21-48.

4. Dougnac A, Santelices L y Chávez A. Tutoría y Razonamiento Clínico. Santiago, Chile: Editorila Finis Terrae; 2016. 166p.

5. Sánchez I. The academic career of clinical teachers. Rev Med Chile 2009; 137 (8): 1113-6.

6. Gutiérrez-García C, Pérez-Pueyo Á, Pérez-Gutiérrez M, Palacios-Picos A. Percepciones de profesores y alumnos sobre la enseñanza, evaluación y desarrollo de competencias en estudios universitarios de formación de profesorado. Cult y Educ 2011; 23 (4): 499-514.

7. Mundina JB, Pombo MNC, Ruiz ER. La docencia y la tutoría en el nuevo marco universitario. Revista electrónica interuniversitaria de formación del profesorado 2005; 8 (5): 12.

8. Vizcaya MF, Pérez RM, Jurado J, Cibanal L, Domínguez JM, Gabaldón EM, Cartagena de la Peña E, Aragonés LAM, Fernández P, Lillo M, Sanjuán A (2006). Experiencia de Acción Tutorial Virtual en la Docencia Práctica Clínica en los Estudios de Enfermería. Disponible en: http://www.academia.edu/469338/Experiencia_de_Accion_Tutorial_Virtual_en_la_Docencia_Practica_Clinica_en (acceso: 9 de diciembre de 2019).

9. Moleiro L, Torres JA. Metodología para el perfeccionamiento de los tutores de formación de los estudiantes de pregrado. Revista EDUMECENTRO 2013; 5 (2): 103-15.

10. Flores-Hernández F, Gatica-Lara F, Sánchez-Mendiola
M, Martínez-González A. Evolución de la evaluación del desempeño docente en la Facultad de Medicina; evidencia de validez y confiabilidad. Inv Ed Med 2017; 6 (22): 96-103.

11. Herskovic P. La formación pedagógica de académicos clínicos en la Facultad de Medicina de la Universidad de Chile. Rev Hosp Clínico Univ Chile 2009; 20: 315-8.

12. Cortés E. El médico como profesor universitario: percepción de un grupo de académicos del Departamento de Medicina del Hospital Clínico de la Universidad de Chile. Rev Hosp Clin Univ Chile. 2009; 20: 319-30.

13. Harden RM, Crosby J. AMEE Guide No 20: The good teacher is more than a lecturer - the twelve roles of the teacher. Medical Teacher 2000; 22 (4): 334-47.

14. Hesketh EA, Bagnall G, Buckley EG, Friedman M, Goodall E, Harden RM, et al. A framework for developing excellence as a clinical educator. Medical Education 2001; 35 (6): 555-364.

15. Ramani S. S, Leinster SS. S. AMEE Guide no.34: Teaching in the clinical environment. Medical Teacher 2008; 30 (34): 347-64.

16. McLean M, Cilliers F, Van Wyk JM. Faculty development: Yesterday, today and tomorrow. Medical Teacher 2008; 30 (6): 555-84.

17. Triviño X, Sirhan M, Moore P, Reyes C. Formación en educación de los docentes clínicos de medicina. Rev Med Chile 2009; 137 (11): 1516-22.

18. Giorgi A. Sketch of a psychological phenomenological method. In: Aanstoos CM; Fischer WF; Giorgi A; Wertz F.J; eds. Phenomenology and psychological research. Pittsburgh: Duquesne University Press 1985; 8-22.

19. Álvarez JA, Vicent MD, Salamanca JM, Pérez F, Miguel I. El tutor y la tutoría en el proceso de formación de especialistas sanitarios en la comunidad de Madrid. Análisis e interpretación mediante grupos focales. Educación médica 2003; 6 (2): 100-11.

20. González R, Cardentey J. Percepción de estudiantes de medicina sobre el desempeño del profesor en el escenario docente. Revista Habanera Ciencias Médicas. 2015; 14 (6): 1-8. 